PAPER

\title{
Eighteen- and 24-month-old infants correct others in anticipation of action mistakes
}

\section{Birgit Knudsen ${ }^{1}$ and Ulf Liszkowski ${ }^{1,2}$}

1. Max Planck Research Group Communication before Language, Max-Planck-Institute for Psycholinguistics, The Netherlands

2. Donders Institute for Brain, Cognition and Behaviour, Radboud University, The Netherlands

\begin{abstract}
Much of human communication and collaboration is predicated on making predictions about others' actions. Humans frequently use predictions about others' action mistakes to correct others and spare them mistakes. Such anticipatory correcting reveals a social motivation for unsolicited helping. Cognitively, it requires forward inferences about others' actions through mental attributions of goal and reality representations. The current study shows that infants spontaneously intervene when an adult is mistaken about the location of an object she is about to retrieve. Infants pointed out a correct location for an adult before she was about to commit a mistake. Infants did not intervene in control conditions when the adult had witnessed the misplacement, or when she did not intend to retrieve the misplaced object. Results suggest that preverbal infants anticipate a person's mistaken action through mental attributions of both her goal and reality representations, and correct her proactively by spontaneously providing unsolicited information.
\end{abstract}

\section{Introduction}

Much human communication and collaboration is predicated on making predictions about others' actions. Predicting others' actions enables one to interact rapidly and in accordance with others' actions before these are executed. Many animals exploit others' actions for their own benefit, as it were with Machiavellian-like intelligence (Byrne \& Whiten, 1988; Hare \& Tomasello, 2004). However, humans use their action predictions also to help others avoid mistakes and mishaps. For example, if you see your friend parking her car in the parking fee zone in front of your house you may anticipate her going to the parking meter to buy a ticket. However, if you know that the parking meter broke that very morning, you might spontaneously help her and indicate another parking meter to spare her walking to the broken one and losing money. The current paper investigates the ontogenetic roots of what we call 'anticipatory correcting' and asks when children begin to intervene helpfully and correct a person in anticipation of her acting mistakenly, that is, before she initiates an action that will lead to a mistake or mishap.

Predicting others' actions is based on 'theory-of-mind' skills (Premack \& Woodruff, 1978) which are most evident in the case of predicting others' action mistakes (Dennett, 1978). Cognitively, the prediction of action mistakes requires both an understanding of a person's goal prior to her acting ('prior intentions'; Searle, 1983; e.g. getting a parking ticket); and an understanding of her incorrect representation of reality that will lead her to act mistakenly (e.g. going to a broken parking meter). Both of these mental attributions are necessary and neither of them alone is sufficient to predict a mistaken action. In addition, predicting a goal-oriented action before it is initiated is based on some form of 'forward' inferences from the actor's mental representations to her action goal. This is different from the case of observing and making sense of completed or ongoing goal-oriented actions, which is based on 'backward' inferences from the observed goal-oriented action to the actor's mental representations (e.g. Bartsch \& Wellman, 1989).

Most infant studies have investigated social-cognitive attributions of goal and reality representations separately (for studies with verbal children, see also Leslie, German $\&$ Polizzi, 2005). For example, infants around their first birthday interpret a completed action in terms of its goal, as revealed by habituation studies (Woodward, 1998; Gergely, Nadasdy, Csibra \& Biro, 1995) and imitation studies (Carpenter, Akhtar \& Tomasello, 1998). But infants also infer the unseen goal of an ongoing action. For example, in action-based imitation studies infants reproduce the unseen goal of a failed action attempt at 18 (Meltzoff, 1995) and 12 months of age (Nielsen, 2006). Further, a recent looking-time study shows that 12-month-olds will look longer at a single chasing event

Address for correspondence: Birgit Knudsen, Max Planck Research Group Communication before Language, Max-Planck-Institute for Psycholinguistics, Wundtlaan 1, 6525 XD Nijmegen, The Netherlands; e-mail: Birgit.Knudsen@mpi.nl 
of two balls when it does not result in a catch than when it does (Southgate \& Csibra, 2009). In other designs using multiple exposures to a goal-directed action, an eye-tracking study shows that 12-month-olds will look ahead of time to a bucket to which a human hand is transferring an object, but not when the object moves self-propelled (Falck-Ytter, Gredebäck \& von Hofsten, 2006). And an EEG study of sensorimotor activation reveals that 9-month-olds are sensitive to others' goals during the online observation of a goal-directed grasping action (Southgate, Johnson, El Karoui \& Csibra, 2010b).

Around 12 months of age infants thus infer a goal when watching an ongoing action that is directed towards an occluded or unreached goal. Presumably, infants infer the unseen goal of an ongoing, goaloriented action through backward reasoning from the ongoing action to the actor's goal representation (Gloss: 'What is she doing? She must be trying XYZ'). It is less clear to what extent infants also make 'forward' predictions from an actor's goal representation to her action before it is directed towards a discernable goal. To do so, infants must know something about another's goal prior to the goal-directed action. An imitation study shows that 2-year-old children indeed consider others' prior intentions when observing an ongoing action demonstration. In that study, 2-year-olds understood the goal of an ongoing action demonstration in terms of what the demonstrator had done or intended to do before she demonstrated the to-be-copied action (Carpenter, Call \& Tomasello, 2002; see also Southgate \& Csibra, 2009). Though based on backward predictions, the study shows that at least by 2 years of age children are sensitive to others' prior intentions, a necessary prerequisite for anticipatory correcting.

However, to predict that a person will act erroneously one also needs to consider the person's epistemic representation of reality. Recent looking-time studies suggest that infants in their second year of life are sensitive to another's epistemic relation to the world when interpreting her ongoing action. Infants will look longer at psychologically implausible events, for example when an actor reaches for an object in one of two locations that is incongruent with her last information about the object's location, whether she obtained the information through visual observation (Onishi \& Baillargeon, 2005; Southgate, Senju \& Csibra, 2007), communication, (Song, Onishi, Baillargeon \& Fisher, 2008) or manual exploration (Träuble, Marinović \& Pauen, 2010; for a recent overview see Baillargeon, Scott \& He, 2010). Since violation-of-expectation studies require recognition of 'after-the-fact incongruent events' (Keen, 2003, cited in Southgate et al., 2007) and are presumably based on backward inferences, Southgate et al. (2007) investigated anticipatory gaze shifts to test whether children also make forward predictions about an actor's action based on her representations of reality. In their eye-tracking study, 25-month-old children were first trained with a discriminative cue to make a gaze shift to one of two doors (the doors lit up and a sound occurred) through which an actor subsequently reached to retrieve an object from a box in front of it. Sixty-one percent of the 2-yearolds succeeded in making prospective gaze shifts at training and test. At test, after the discriminative cue, a significant majority of these children looked at the door through which the actor was about to reach for the object where she had last seen it - even when the object had been moved to the alternative box and was then removed altogether from the scene. The study thus shows that 2year-olds engage in 'forward' reasoning from epistemic states to actions that have not yet been executed. With regard to anticipatory correcting, current research thus suggests that at least each of the two cognitive prerequisites, that is forward inferences about others' actions from goal representations and reality representations, are present around 2 years of age.

Another question, however, is whether infants are able and motivated to use their forward action prediction skills productively to help others avoid prospective mistakes. Previous research has suggested that children use the cognitive skills productively in guiding their own actions only around age 4 when they begin to lie to others (e.g. Talwar \& Lee, 2008) and become sensitive to misinformation (Sperber, Clément, Heintz, Mascaro, Mercier, Origgi \& Wilson, 2010). When lying, one has to consider a person's prior goal and then induce an incorrect representation of reality, in anticipation of this incorrect representation leading the person to pursue her goal incorrectly and thus act mistakenly. But since human communication is truthful and cooperative by default and lying is the exception, it is entirely possible that before higher-order deceptive and competitive motives emerge, children make and use predictions of action mistakes to help others in collaborative interactions.

Recent studies reveal that infants are indeed motivated to help an adult who encounters a problem. For example, in the second year, infants will empathically help an adult who expresses sorrow over the loss of a toy (e.g. a broken teddy) by compensating the loss with a duplicate toy (Bischof-Köhler, 1991). And 18-month-olds will instrumentally help an adult who expresses dissatisfaction and struggles in attaining a goal by assisting in her task (e.g. open a door or box; Warneken \& Tomasello, 2006). Further, 18-month-olds also seem to tailor their instrumental helping to the adult's epistemic relation to his goal. In a recent study (Buttelmann, Carpenter \& Tomasello, 2009), infants were first trained how to open a box. At test, $64 \%$ of the 18 -month-old infants who did not fuss then helped an adult who tried effortfully to open one of two boxes. In one condition, the adult had not witnessed that a toy had been swapped from one to the other box. In that condition, the children who helped in reaction to the adult's difficulty mostly opened the other box to retrieve the toy. The spontaneous helping response was moderately pronounced in that study, given that the behavior had been practiced just before. However, the cognitive interpretation that those infants who 
helped did so by considering the adult's representation of reality has recently been corroborated in a similar study (Southgate, Chevallier \& Csibra, 2010a). In that study, 17 -month-olds had to comply with an adult's request to retrieve one of two objects that were each in a box. First, infants practiced retrieving a toy from a box when the experimenter requested it. At test, when the adult had not witnessed a swap of the objects and now requested an object by pointing to a box, the significant majority of infants retrieved the object from the other box. Although the study did not involve prosocial helping but compliance with a request, it supports the cognitive interpretation of previous looking-time studies and suggests that infants interpret and react appropriately to an adult's mistaken action by reference to her representation of reality.

However, anticipatory correcting is based on the usage of forward predictions and a motivation to help others spontaneously, that is, before help is solicited. One troubling aspect of the previous helping studies is that infants always reacted after a problem or mishap had occurred. Thus, it is possible that infants reacted either to the adult's request for help (possibly as part of general compliance; e.g. Kärtner, Keller \& Chaudhary, 2010), or directly to remedy the aversive situation (e.g. Preston \& de Waal, 2002). However, anticipatory correcting as illustrated in our example requires intervening before any problem has yet occurred. It is possible that this kind of proactive helping is especially common in communicative acts, for example when warning others (Knudsen \& Liszkowski, 2010), or informing them about changes that are relevant to future behaviors. Recent studies have shown that 12-month-olds indeed help an adult who searches for an object by informing her through communicative pointing (Liszkowski, Carpenter, Striano \& Tomasello, 2006), more so when the adult has not seen the object than when she has (Liszkowski, Carpenter \& Tomasello, 2008). However, in these studies, too, the adult was explicitly searching for an object and expressing some kind of puzzlement and frustration, so that it is entirely possible that infants simply complied with the request for information, or in an effort to remedy the problem. It is less clear whether infants would spontaneously help an adult who does not yet have a problem by communicating to him relevant information. This kind of intervening would not only constitute evidence for spontaneous behavioral usage of forward action predictions. It would also corroborate theories of altruism in infancy (Warneken \& Tomasello, 2009).

In the current study we investigated whether 18- and 24-month-old infants would correct an adult communicatively with a non-verbal pointing gesture when she was about to commit an action mistake. In particular we were interested in whether such anticipatory correcting would occur fully spontaneously and be based on an integration of both the adult's goal representation and her representation of reality. We designed an interactive paradigm and manipulated both of these factors independently. To manipulate the adult's prior intention, infants first watched an adult either enact her intention to find an object in a set of containers (correct action condition; mistaken action condition) or clean these containers (different action condition). In both cases she found an object and then left the scene. To manipulate the adult's representation of reality, a second experimenter subsequently changed the location of the object either in the absence (mistaken action condition; different action condition) or presence (correct action condition) of the adult. At test, the adult re-entered without indicating which container she would approach.

Based on the reviewed research we expected that infants would be motivated to help and intervene spontaneously to correct the adult before she acted mistakenly or displayed any signs of discomfort or problems. We expected that infants would intervene selectively by integrating both her goal representation and her representation of reality. We thus predicted that infants would point to the object's new location only when the adult intended to retrieve the object but had an incorrect representation of its location (mistaken action condition). In contrast, infants should not point to the object's new location in the different action condition, when the adult had an incorrect representation of the object's location but pursued a different goal. And infants should not point to the object's new location in the correct action condition, when the adult had a correct representation of the object's location and intended to retrieve it. Since we were interested in infants' truly spontaneous, actionbased usage of action predictions, we did not administer any pre-training on pointing, informing, helping, or any other additional inclusion criteria. In addition, since we wanted to investigate 'forward' action predictions we were careful to test infants' behavior while the adult's action was still ambiguous, that is before it was apparent in the adult's orienting and reaching behavior on which container she would act.

\section{Method}

\section{Participants}

Forty-eight 18-month-olds ( 24 girls, 24 boys; $M=18 ; 10$, range $=17 ; 28-18 ; 27$ ) and 16 24-month-olds (eight girls, eight boys; $M=24 ; 14$, range $=24 ; 9-24 ; 28$ ) participated in the study. Infants were recruited from a database of parents who volunteered to participate in infancy research. Seven 18-month-olds and six 24-month-olds were excluded because of fussiness (10), experimenter error (two), or parental interference (one).

\section{Set-up and materials}

The testing room measured $4.60 \times 4.60 \mathrm{~m}$ (see Figure 1). Eighty centimetres to the side of the table a room-divider partitioned off $1 \mathrm{~m}$ of the room. Two cameras recorded the infant and two cameras recorded the experimenter. 

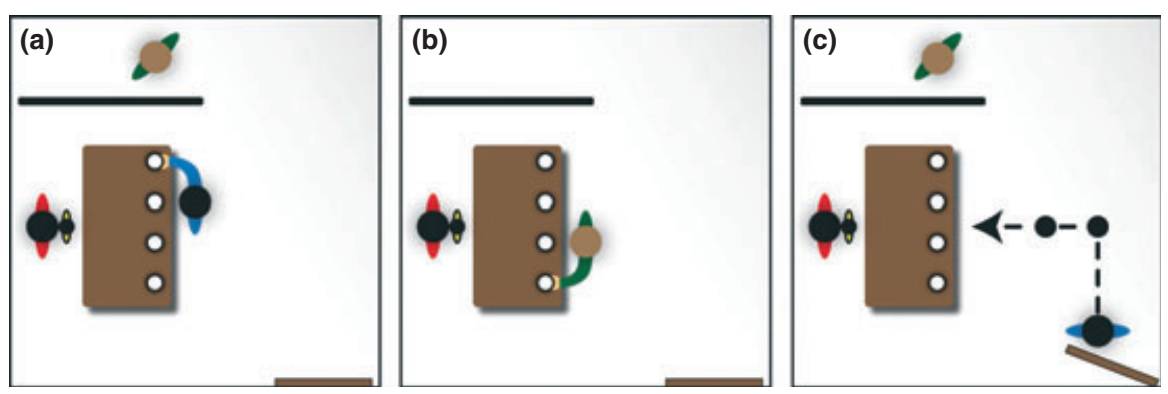

Figure 1 Schematic depiction of the set-up and procedure. (a): Context phase. E1 either searches for an object which she had found in the last container (mistaken action and correct action conditions) or cleans the containers and accidentally finds the object in the last container (different action condition). E2 is hiding behind a room-divider. (b): Switch phase. E2 places the object into the container on the opposite side, either unbeknown to E1 (mistaken action and different action conditions) or while E1 is watching (correct action condition; not depicted here). (c): Test. E1 enters the room and walks in a predefined L-shaped path, first in parallel to the table $(2 \mathrm{~m})$, then slowly approaching the table $(2 \mathrm{~m})$.

Two sets of four identical opaque containers were used. They were equally spaced across the table on E1's side, approximately $70 \mathrm{~cm}$ away from the infant. For the 24-month-olds an additional two sets of containers were used. One novel object was used in each trial. For the 18-month-olds these were (i) a white plastic lid with yellow tape and (ii) a piece of sponge. For the 24-montholds, two additional objects were (iii) an O-shaped and (iv) a trapeze-shaped curtain holder.

\section{Procedure}

Infants sat on their parent's lap at a table facing the experimenter (E1). A second experimenter (E2) hid behind the room-divider. Parents were instructed to remain silent and not to interfere. The 18-month-olds were randomly assigned to one of three conditions. Each condition consisted of two trials which were separated by brief play. Each trial consisted of three phases. Each trial began with E1 lining up the four containers of a set on her side of the table. The order of container sets was counterbalanced across participants. Either the far left or the far right container contained one of the novel objects. The order alternated and was fully counterbalanced.

\section{Context phase}

In the mistaken action and correct action conditions, E1 searched for the object. She opened the first container, showed it to the infant and said with mild disappointment: 'No, it's not in here. Hmm, where could it be?' She proceeded in the exact same manner with the other containers. When she found the object in the last container, she said excitedly: 'Hey! Here it is! That's neat!' In the different action condition, instead of searching, E1 cleaned the containers and then attached a sticker. She opened the first container, showed it to the infant and said: 'I'm cleaning it'. She wiped out the container and said 'Look what I'm doing' and she marked the container with a small sticker to signal that she had finished. When she found the object in the last container she acted with surprise and said: 'Hey! There's an object! That's a surprise!' In all three conditions, E1 then held up the object, pretended that someone had knocked at the door, and said that she had to leave quickly. She put the object back into the container making sure infants watched, stood up, tapped on the container and said she would be right back to continue.

\section{Switch phase}

E2 appeared from behind the room-divider in a slightly conspicuous manner, greeted the infant and took the object out of the container, held it up and said ' $\mathrm{Oh}$, look'. She alternated gaze between the door and the infant and said 'Look! I'm putting it in here'. She put the object inside the container on the opposite side, alternated gaze between the door and the infant, showed the object again and placed it back into the new container. Then she hid again behind the room-divider and E1 re-entered the room. In the correct action condition, E1 re-entered the room earlier, stood at the door, and E1 and E2 greeted each other in a friendly manner saying 'hello'. When E2 retrieved the toy she also called E1 and said '[E1's name], look!'. When E2 put the object into the new container she also said to E1, 'Look [E1's name], I'm putting it in here'. E1 took two steps towards the table to demonstrate that she witnessed where the toy was going, and said 'Ah, OK!', alternating gaze between the new container and the infant. Then, E1 stepped back again to close the door and waited there to begin the test phase. E1 and E2 said goodbye to each other and E2 hid behind the room-divider.

\section{Test}

The test phase took about 30 seconds and began with E1 standing at the door. In a predetermined and timed sequence she first said, 'Good. Shall we continue playing?' and then approached the table steadily on an L-shaped path, walking confidently but never indicating which box she would approach, stopping twice on two 
predefined spots (see Figure 1). She first walked about 2 meters in parallel to the table, stopped briefly, looked at the infant and said 'Okay, let's go on', and then turned to approach the table perpendicularly for another 2 meters, stopped again, looked at the infant and said again 'Okay, let's go on'. The test phase ended as soon as E1 had sat down and before she lifted her arms to reach for one of the containers. If the infant pointed during this period, E1 looked at the infant and said 'Yes', but continued her predetermined and timed sequence. Only data obtained during this anticipation period were analyzed. Trials ended with E1 acting on the container congruent with her goal and reality representation by either putting a sticker on the empty container (different action condition), or retrieving the toy (correct action condition). In the mistaken action condition, if infants had not pointed, E1 first reached for and opened the now-empty box, showed it to the infant and said 'Huh? How is this possible? It is empty!' (Search phase 1). If infants did not point, E1 continued 'Where is the toy? [Name of infant], do you know where the toy is?' (Search phase 2). If infants had not pointed, E1 always found the object in the course of putting away the containers and said 'Ah, look! Here it is!'

The 24-month-olds were tested after completion of the study with the 18-month-olds in an attempt to test for an increase in performance in the mistaken action condition and to replicate findings in a within-subject design. Since we expected that infants would become restless after 4 trials, we administered only the mistaken action and correct action conditions with order fully counterbalanced.

\section{Coding and reliability}

Index-finger pointing was coded by the first author if it occurred during the test phase with the arm and indexfinger either fully or half extended towards the current or previous locations of the object, or the room-divider. None of the points were addressed to the mothers who sat still behind their infants. A second coder who was naive to the hypotheses of the study recoded infants' number of points in each of the two test phases of $28 \%$ of the infants in each condition. Inter-rater reliability was excellent, Cohen's Kappa $=.92$ for the 18-month-olds and Cohen's Kappa $=.88$ for the 24-month-olds. A manipulation check on the test phase was conducted by a third coder who was unaware of the purpose of the study. Videos of 15 18-month-olds and five 24-month-olds were randomly selected and cut to the test phases. The coder judged in a two-alternative forced choice task whether E1 would approach the left or right container. For both age groups the coder was at chance in predicting the container (18-months-olds, binomial test, $p=.362$; 24months-olds, binomial test, $p=.824$ ) with no significant differences between conditions (at each age respectively, $\left.\chi^{2}(n=30)=.659 ; \chi^{2}(n=20)=.370\right)$.

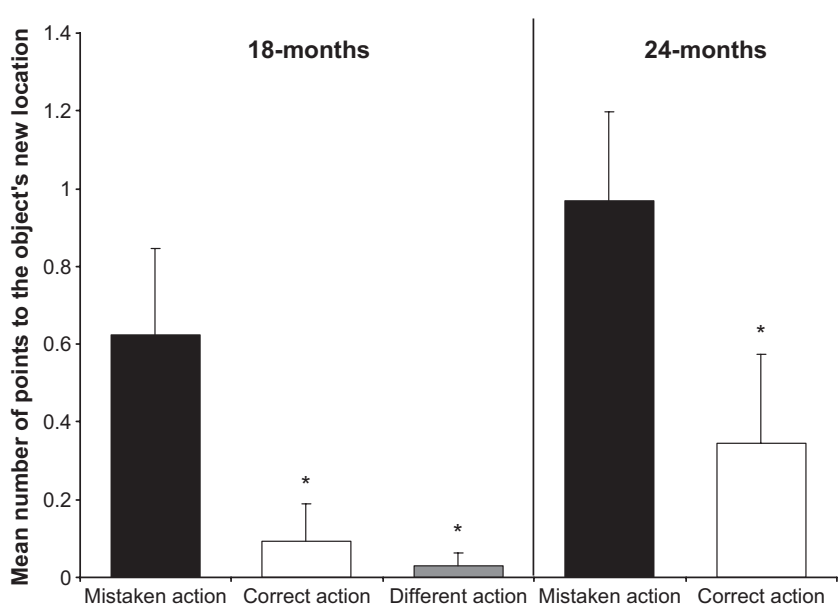

Figure 2 Mean number of points per trial to the target container. Eighteen-month-olds were tested in a between-subjects design (left panel), 24-month-olds in a within-subject design (right panel). Asterisks indicate significant differences.

\section{Results}

The left panel of Figure 2 shows significant differences in the mean number of points across conditions, $F(2$, $45)=5.44, p=.008$. Following our predictions, planned LSD post-hoc tests (one-tailed, based on the pooled standard deviation of the ANOVA) revealed that 18month-olds pointed significantly more to the object's new location in the mistaken action condition compared to the correct action or different action conditions, respectively; mean difference $=.531, p=.005$, Cohen's $d=.782$ and mean difference $=.594, p=.002$, Cohen's $d=.939$. The left panel of Figure 3 shows that on the individual level significantly more 18 -month-olds pointed at least once to the new object's location in the mistaken action $(7 / 16)$ than correct action $(1 / 16)$ or

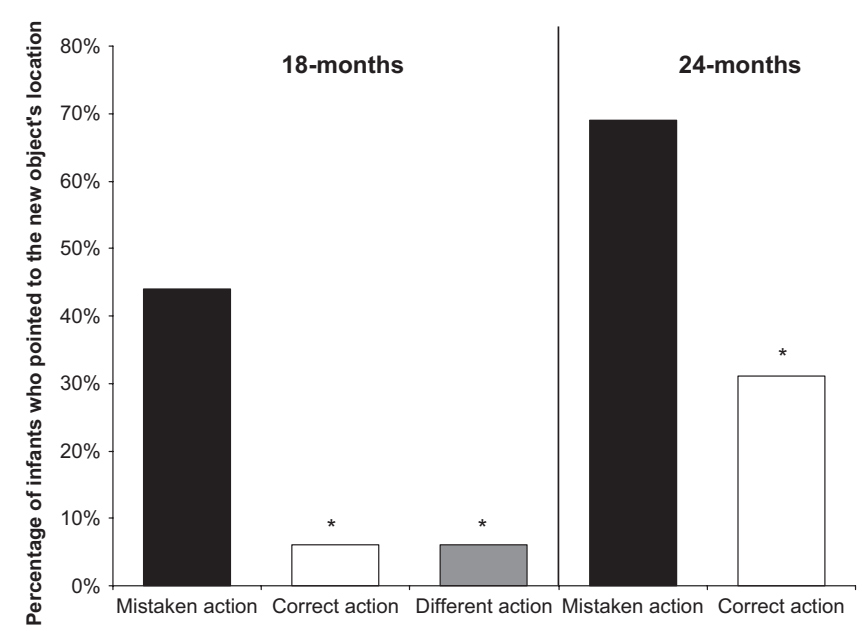

Figure 3 Percentage of infants who pointed at least once. Eighteen-month-olds were tested in a between-subjects design (left panel), 24-month-olds in a within-subject design (right panel). Asterisks indicate significant differences. 
different action (1/16) conditions (Fisher's Exact tests, both $p \mathrm{~s}=.019)$. There were no significant differences between first and second trial performance, neither with regard to the mean number of points (Wilcoxon, $p=.931)$ nor with regard to the number of infants who pointed (McNemar, $p=1)$. Although there were no differences across the two trials, when looking at the first trial only, the pattern of individual performance remained similar. It approached significance between the mistaken action and correct action conditions (Fisher's Exact tests, $p=.086$ ), and was significant between the mistaken action and different action conditions (Fisher's Exact tests, $p=.022$ ).

Additional analyses revealed little pointing to the object's previous location (mistaken action condition, $M=.156, S D=.507$; correct action condition, $M=$ .000 ; different action condition, $M=.094, S D=.272$ ) or the second adult's hiding place (mistaken action and correct action condition, $M=.031, S D=.125$; different action condition, $M=.344, S D=.889$ ), and no systematic differences between the conditions, thus further excluding alternative possibilities of what infants were doing when pointing. Finally, in the mistaken action condition, after E1 had reached for and opened the nowempty box, three additional infants pointed to the other (target) box (totaling $62.5 \%$ of all infants; two pointed in the first trial of search phase 1; one in the second trial of search phase 2).

At 24 months, more than half of the infants exhibited the target behavior in the mistaken action condition. Results were replicated in a within-subject design; see right-hand panels of Figures 2 and 3, for both number of points $(t(15)=3.72, p=.001$, Cohen's $d=.794)$ and number of infants (McNemar, $p=.035$ ). Further, in the mistaken action condition, after E1 had reached for and opened the now-empty box, two additional infants pointed to the other (target) box (totalling $81 \%$ of all infants; both pointed in search phase 1).

\section{Discussion}

Infants used a non-verbal pointing gesture to correct an adult who was about to - but had not yet started to - act mistakenly and retrieve an object from a wrong location. Infants corrected the adult selectively depending on the adult's prior goal representation and her representation of reality. Such a form of anticipatory correcting reveals an early manifestation of an unsolicited and spontaneous behavioral usage of forward action predictions. The fact that infants helped even before an aversive situation, problem or request for help had occurred underscores infants' prosocial concern for others.

Infants pointed selectively in the mistaken action condition, when the adult had the goal of retrieving the object but an incorrect representation about its location. Importantly, infants provided the new information only when it was relevant to the combination of both the adult's prior goal representation and her representation of reality. When either of these factors was altered separately, for example when the adult had not witnessed the location change but intended to continue cleaning the container (different action condition), or when the adult intended to retrieve the object and had already witnessed the location change (correct action condition), infants did not point for the adult. Thus, infants integrated both goal and reality representations in generating predictions about the adult's next action. The integration of these two directions-of-fit (Searle, 1983) is a core aspect in the emergence of desire-belief psychology and theory of mind (e.g. Wellman, 1990).

At the same time the pattern of results excludes several alternative possibilities of what infants were doing when pointing. For example, if infants always pointed out for the adult what was new (e.g. Liszkowski, Carpenter \& Tomasello, 2007) or what she had not witnessed (O'Neill, 1996), they should have pointed in both of the conditions in which the adult did not witness the location change. And they should have pointed to the second adult's hiding place when the adult had not seen her. Alternatively, if infants always wanted to comment on or request the next step in an action sequence, infants should have pointed to the object's new location irrespective of whether the adult had or had not witnessed the location change. Further, in the different action condition they should have pointed to the unmarked container where the action should continue. Finally, if infants pointed only egocentrically, they should have pointed in all three conditions equally, for example to request the object for themselves, or for other, non-communicative reasons. Instead, infants pointed only in the mistaken action condition, which is in accordance with the interpretation that infants corrected the adult in anticipation of her committing a mistake.

Infants' pointing was spontaneous and unsolicited, without prior training, before an action mistake of another person had happened. Previous looking-time studies have revealed infants' understanding of actions by measuring spontaneous increase of visual attention in response to erroneous actions (see Baillargeon et al., 2010), or by measuring anticipatory looking in cued choices about an expected action (at 2 years of age; Southgate et al., 2007). These studies reveal infants' information processing capacities (respectively, backward and forward inferences about others' mistaken actions). However, they did not address infants' behavioral usage of these cognitive skills. It is important to note that in the current study we neither excluded participants who did not show the target behavior, nor did we train participants to produce the target behavior, as has been the case in previous studies. For example, in the Buttelmann et al. (2009) study, the percentage of 18month-olds who helped freely $(64 \%)$ may be more comparable to our 24- than 18-month-olds. However, in that study (Buttelmann et al., 2009; see also Southgate et al., 2010a), participants also received prior training on the 
target behavior. In the current study infants were not trained to point for the adult. It is possible that familiarizing infants with a searching and questioning adult beforehand, and engaging them in pointing activities, would have increased infants' pointing at test. However, this would have precluded measuring spontaneous behavior.

Another difference lies in the directionality of the predictions infants had to make in order to tailor their actions accordingly. In the previous action-based studies (Buttelmann et al., 2009; Southgate et al., 2010a; Carpenter et al., 2002), infants presumably had to go through an inference of the kind 'she is doing $\mathrm{X}$ because she intends/believes Y'. In the current study, to correct someone in anticipation of an action mistake, infants presumably had to make an inference in the opposite direction: 'she intends/believes $\mathrm{X}$ and so will do $\mathrm{Y}$ '. For older, verbal children it has been suggested that the latter kind of 'forward reasoning' from others' goals and beliefs to their actions apparently emerges after 'backward reasoning' from others' actions to their goals and beliefs (Bartsch \& Wellman, 1989; Robinson \& Mitchell, 1995). When including infants' performance in the current study after the anticipation phase, that is when the adult was already acting mistakenly or expressing problems, target performance increased to $62.5 \%$ at 18 months (comparable to Buttelmann et al., 2009), and $81 \%$ at 24 months.

Beginning around 18 months of age (as conventionally indicated by the median age of emergence; e.g. Carpenter, Nagell \& Tomasello, 1998), and developing throughout the second year, infants use forward inferences about a person's next action to spontaneously guide their own, uncued and unsolicited actions. Although results of the first trial only approached significance for one comparison for the 18-month-olds, it is more likely that this reflects reduced statistical power than that infants' performance was based on procedural feedback of the first trial. First, there were no statistical differences in infants' performance between the two trials. Second, if anything, infants might have learned that the adult retrieved the object in the mistaken action condition anyway, which should have led them to point less in the second trial. The quite large effect sizes and the within-subject replication at 24 months of age confirm the reliable and selective occurrence of the target behavior. The results suggest an increase in infants' anticipatory correcting over the second half of the second year of life, which is corroborated by Southgate et al.'s (2007) finding of predictive 'theory-of-mind' processing at 25 months of age. The developmental increase may reflect infants' increasing communicative competencies and experiences over the second year of life. Post-hoc inspections of the video recordings further revealed that some 24 -month-olds (63\%) also verbalized about the new object's location. The number of verbalizations about the target container, for example 'Look here' or 'In there', were more frequent in the mistaken action condition
$(72 \%)$ than in the correct action condition (28\%), thus further supporting the interpretation that infants were communicating to the adult about the correct location of the object to spare her making a mistake.

Recent research has revitalized the question whether chimpanzees (Call \& Tomasello, 2008) and infants (Baillargeon et al., 2010) have a theory of mind. While traditional accounts accept convincing evidence for a representational theory of mind only from explicit, usually verbal false belief tasks (Perner, 1991; Carpendale \& Lewis, 2004), other researchers have criticized the additional task demands and have suggested abandoning the classic false belief task (Bloom \& German, 2000; Roth \& Leslie, 1998; Birch \& Bloom, 2007). New implicit, looking-based tasks no longer entail these additional demands like language and choice, but researchers have usually maintained an interpretation in terms of 'false belief'. This interpretation has not remained uncontested. For example, some researchers have suggested two different theory-of-mind systems (Apperly \& Butterfill, 2009), possibly operating on implicit versus explicit, or automatic versus conscious levels, the former being present in infancy, the latter developing well beyond the preschool period. Other alternatives to 'false belief'based interpretations are that children expect a person to look for her object where she last saw or touched it (e.g. Perner \& Ruffman, 2005), or attribute a lack of, rather than an incorrect representation of, reality (e.g. Southgate et al., 2007; Wellmann, 2010). Most infancy studies to date acknowledge the possibility of an alternative interpretation of their data in terms of behavioral rules or ignorance (e.g. Onishi \& Baillargeon, 2005, p. 257; Baillargeon et al., 2010, p. 33; Surian, Caldi \& Sperber, 2007, p. 583; Southgate et al., 2007, p. 590; Southgate et al., 2010a, p. 911; Buttelmann et al., 2009, p. 341; Träuble et al., 2010, p. 436), which suggests that the precise cognitive mechanisms of 'theory of mind' in infancy are not yet fully understood.

The current study investigated whether infants spontaneously use theory-of-mind skills in guiding their own, unsolicited acting. We cannot rule out that infants' communicative actions were based on a general expectation that a person goes to where she last saw an object, a general alternative interpretation for all changeof-location tasks. It is also at least possible that infants expected the person to make a mistake by attributing to her a lack of representation, rather than an incorrect representation of reality. The latter account holds that children assume that when a person doesn't know where an object is she will always err instead of getting it sometimes right by chance. However, studies pitting chance against error do not support this proposal in adults and children (Friedman \& Petrashek, 2009) and in 18-month-olds (Scott \& Baillargeon, 2009). Further, other studies have shown that infants keep track over time of what other people know (Moll, Richter, Carpenter \& Tomasello, 2008; Saylor \& Ganea, 2007). It is reasonable to assume that infants kept track of what the 
adult knew also in the mistaken action condition and thus expected her to search in the container in which she had last seen the object. It is also important to note that the adult did not express any uncertainty or other signs of ignorance or search behavior as in previous studies (Liszkowski et al., 2006, 2008).

The current study neither tested infants' (visual) social-cognitive processing nor children's (verbal) elaborations and theorizing about others' actions. Instead, it advanced a usage-based approach. Infants could freely choose to act or not in accordance with another's to-be-expected action, while the criteria for the kind of required forward action prediction were those typically used in verbal 'theory-of-mind' tasks with older children. Although even those verbal tasks have remained amenable to alternative rule-based interpretations, one argument in favor of a 'false belief' interpretation of those tasks is that children also exhibit spontaneous false belief-based behaviors in a variety of other real-life situations when communicating and interacting with others. Similarly, to understand the scope of infant 'theory of mind', it is crucial to understand whether and how infants use their putative social-cognitive skills.

Previous research has shown that verbal children productively use forward predictions from goal and reality representations to others' mistaken actions when they begin to lie at around age 4. In the current study, infants were given the chance to help an otherwise unfamiliar person in an unfamiliar setting. Helping is a primary aspect of human cooperative communication that logically precedes higher-order deceptive motives. Importantly, infants helped before the person even knew that she would need information. This finding provides new support for theories of altruism in infancy. In extension to previous findings (see Warneken \& Tomasello, 2009), it is clear from the current design that infants did not simply react to an adult's expression of an emotional, instrumental or epistemic problem, a request for help, or in an attempt to directly remedy an aversive situation. Instead they helped proactively on the mental basis of an anticipated, not yet executed, action mistake. The type of proactive helping as revealed by anticipatory correcting is presumably mostly used for communication and is primarily based on social-cognitive rather than empathic and sympathetic processes (for the latter, see Vaish, Carpenter \& Tomasello, 2009). First, through communication one can coordinate with relatively little effort others' actions towards a shared goal and so achieve well-coordinated collaboration. Second, communication itself is a shared activity. Conversations require rapid predictions of others' turns in order to initiate one's own communicative act accordingly (de Ruiter, Mitterer \& Enfield, 2006; Stivers, Enfield, Brown, Englert, Hayashi, Heinemann, Hoymann, Rossano, de Ruiter, Yoon \& Levinson, 2009). Conversations also require helping and correcting each other in maintaining a shared focus and common ground (e.g. Clark, 1996).
The usage-based approach of the current study reveals that infants are motivated to use action predictions productively in the service of unsolicited helpful collaborative interactions several years before they begin to exploit their understanding to misinform and lie to others in a competitive situation. Two other action-based studies (Buttelmann et al., 2009; Southgate et al., 2010a) employed indirectly elicited response tasks and revealed that infants react to another's erroneous action appropriately by helping or complying with a request, presumably through inferences from the ongoing goaloriented action to the actor's misrepresentation of reality. The current study employed an elicitation-free actionbased task and reveals that infants also spontaneously prevent others from committing a mistake, even before the respective object-oriented action is initiated, presumably through inferences from the actor's goal and reality representations of her expected action. Infants use these forward action predictions spontaneously and helpfully to communicate with others. We speculate that infants' behavioral usage of forward predictive 'theory-of-mind' skills emerges through, and is first put to service in, uniquely human forms of collaborative communication.

\section{Acknowledgements}

Our thanks to Margret van Beuningen, Marloes van der Goot, Stefanie Voigt, Nick Wood, Ludy Cilissen (for data collection and coding), Juan-Carlos Gomez, Susan Schmidt (for inspiring discussions).

\section{References}

Apperly, I.A., \& Butterfill, S.A (2009). Do humans have two systems to track beliefs and belief-like states? Psychological Review, 116 (4), 953-970.

Baillargeon, R., Scott, R.M., \& He, Z. (2010). False-belief understanding in infants. Trends in Cognitive Sciences, 14, $110-118$.

Bartsch, K., \& Wellman, H. (1989). Young children's attribution of action to beliefs and desires. Child Development, 60 , 946-964.

Birch, S.A.J., \& Bloom, P. (2007). The curse of knowledge in reasoning about false beliefs. Psychological Science, 18 (5), 382-386.

Bischof-Köhler, D. (1991). The development of empathy in infants. In M.E. Lamb \& H. Keller (Eds.), Infant development: Perspectives from German speaking countries (pp. 245 273). Hillsdale, NJ: Lawrence Erlbaum Associates.

Bloom, P., \& German, T.P. (2000). Two reasons to abandon the false belief task as a test of theory of mind. Cognition, 77 (1), 25-31.

Buttelmann, D., Carpenter, M., \& Tomasello, M. (2009). Eighteen-month-old infants show false belief understanding in an active helping paradigm. Cognition, 112, 337-342.

Byrne, R.W., \& Whiten, A. (1988). Machiavellian intelligence: Social expertise and the evolution of intellect in monkeys, apes, and humans. Oxford: Oxford University Press. 
Call, J., \& Tomasello, M. (2008). Does the chimpanzee have a theory of mind? 30 years later. Trends in Cognitive Sciences, 12, 187-192.

Carpendale, J.E.M., \& Lewis, C. (2004). Constructing an understanding of mind: the development of children's understanding of mind within social interaction. Behavioral and Brain Sciences, 27, 79-150.

Carpenter, M., Akhtar, N., \& Tomasello, M. (1998a). Fourteen- to 18-month-old infants differentially imitate intentional and accidental actions. Infant Behavior and Development, 21, 315-330.

Carpenter, M., Call, J., \& Tomasello, M. (2002). Understanding 'prior intentions' enables 2-year-olds to imitatively learn a complex task. Child Development, 73, 1431-1441.

Carpenter, M., Nagell, K., \& Tomasello, M. (1998b). Social cognition, joint attention, and communicative competence from 9 to 15 months of age. Monographs of the Society for Research in Child Development, 63 (4, Serial No. 255).

Clark, H.H. (1996). Using language. Cambridge: Cambridge University Press.

Dennett, D.C. (1978). Beliefs about beliefs. Behavioral Brain Sciences, 1 (4), 568-570.

de Ruiter, J.P., Mitterer, H., \& Enfield, N.J. (2006). Projecting the end of a speaker's turn: a cognitive cornerstone of conversation. Language, 82 (3), 515-535.

Falck-Ytter T., Gredebäck G., \& von Hofsten C. (2006). Infants predict other people's action goals. Nature Neuroscience, 9 (7), 878-879.

Friedman, O., \& Petrashek, A.R. (2009). Children do not follow the rule 'ignorance means getting it wrong'. Journal of Experimental Child Psychology, 102, 114-121.

Gergely, G., Nadasdy, Z., Csibra, G., \& Biro, S. (1995). Taking the intentional stance at 12 months of age. Cognition, 56, 165-193.

Hare, B., \& Tomasello, M. (2004). Chimpanzees are more skillful in competitive than in cooperative cognitive tasks. Animal Behaviour, 68, 571-581.

Kärtner, J., Keller, H., \& Chaudhary, N. (2010). Cognitive and social influences on early prosocial behavior in two socio-cultural contexts. Developmental Psychology, 46 (4), 905-914.

Keen, R. (2003). Representation of objects and events: why do infants look so smart and toddlers look so dumb? Current Directions in Psychological Science, 12, 79-83.

Knudsen, B., \& Liszkowski, U. (2010). 18-month-old infants warn others in anticipation of negative action effects. Poster presented at 17 th Biennial International Conference on Infant Studies, Baltimore, MD.

Leslie, A.M., German, T.P., \& Polizzi, P. (2005). Belief-desire reasoning as a process of selection. Cognitive Psychology, 50, 45-85.

Liszkowski, U., Carpenter, M., Striano, T., \& Tomasello, M. (2006). Twelve- and 18-month-olds point to provide information for others. Journal of Cognition and Development, 7 (2), 173-187.

Liszkowski, U., Carpenter, M., \& Tomasello, M. (2007). Pointing out new news, old news, and absent referents at 12 months of age. Developmental Science, 10 (2), F1-F7.

Liszkowski, U., Carpenter, M., \& Tomasello, M. (2008). Twelve-month-olds communicate helpfully and appropriately for knowledgeable and ignorant partners. Cognition, 108 (3), $732-739$.
Meltzoff, A. (1995). Understanding the intentions of others: reenactment of intended acts by 18-month-old children. Developmental Psychology, 31, 838-850.

Moll, H., Richter, N., Carpenter, M., \& Tomasello, M. (2008). Fourteen-month-olds know what 'we' have shared in a special way. Infancy, 13 (1), 90-101.

Nielsen, M. (2006). Copying actions and copying outcomes: social learning through the second year. Developmental Psychology, 42, 555-565.

O’Neill, D.K. (1996). Two-year-old children's sensitivity to a parent's knowledge state when making requests. Child Development, 67 (2), 659-677.

Onishi, K.H., \& Baillargeon, R. (2005). Do 15-month-old infants understand false beliefs? Science, 308, 255-258.

Perner, J. (1991). Understanding the representational mind. Cambridge, MA: MIT Press.

Perner, J., \& Ruffman, T. (2005). Infants' insight into the mind: how deep? Science, 308, 214-216.

Premack, D., \& Woodruff, G. (1978). Does the chimpanzee have a theory of mind? Behavioral Brain Sciences, 1 (4), 515526.

Preston, S.D., \& de Waal, F.B.M. (2002). Empathy: its ultimate and proximate bases. Behavioral and Brain Sciences, 25 (1), $1-71$.

Robinson, E.J., \& Mitchell, P. (1995). Masking of children's early understanding of the representational mind: backwards explanation versus prediction. Child Development, 66, 1022 1039.

Roth, D., \& Leslie, A.M. (1998). Solving belief problems: toward a task analysis. Cognition, 66, 1-31.

Saylor, M.M., \& Ganea, P.A. (2007). Infants interpret ambiguous requests for absent objects. Developmental Psychology, 43 (3), 696-704.

Scott, R.M., \& Baillargeon, R. (2009). Which penguin is this? Attributing false beliefs about object identity at 18 months. Child Development, 80, 1172-1196.

Searle, J.R. (1983). Intentionality: An essay in the philosophy of mind. Cambridge: Cambridge University Press.

Song, H., Onishi, K.H., Baillargeon, R., \& Fisher, C. (2008). Can an agent's false belief be corrected by an appropriate communication? Psychological reasoning in 18-month-old infants. Cognition, 109, 295-315.

Southgate, V., Chevallier, C., \& Csibra, G. (2010a). Seventeenmonth-olds appeal to false beliefs to interpret others' referential communication. Developmental Science, 13 (6), 907-912.

Southgate, V., \& Csibra, G. (2009). Inferring the outcome of an ongoing novel action at 13 months. Developmental Psychology, 45, 1794-1798.

Southgate, V., Johnson, M.H., El Karoui, I., \& Csibra, G. (2010b). Motor system activation reveals infants' on-line prediction of others' goals. Psychological Science, 21, 355-359.

Southgate, V., Senju, A., \& Csibra, G. (2007). Action anticipation through attribution of false belief by 2-year-olds. Psychological Science, 18, 587-592.

Sperber, D., Clément, F., Heintz, C., Mascaro, O., Mercier, H., Origgi, G., \& Wilson, D. (2010). Epistemic vigilance. Mind \& Language, 25 (4), 359-393.

Stivers, T., Enfield, N.J., Brown, P., Englert, C., Hayashi, M., Heinemann, T., Hoymann, G., Rossano, F., de Ruiter, J.P., Yoon, K.-E., \& Levinson, S.C. (2009). Universals and cultural variation in turn-taking in conversation. Proceedings of the National Academy of Sciences, USA, 106 (26), 10587-10592. 
Surian, L., Caldi, S., \& Sperber, D. (2007). Attribution of beliefs by 13-month-old infants. Psychological Science, 18, 580586.

Talwar, V., \& Lee, K. (2008). Social and cognitive correlates of children's lying behavior. Child Development, 79, 866-881.

Träuble, B., Marinović, V., \& Pauen, S. (2010). Early theory of mind competencies: do infants understand others' beliefs? Infancy, 15, 434- 444.

Vaish, A., Carpenter, M., \& Tomasello, M. (2009). Sympathy through affective perspective taking and its relation to prosocial behavior in toddlers. Developmental Psychology, 45 (2), 534-543.

Warneken, F., \& Tomasello, M. (2006). Altruistic helping in human infants and young chimpanzees. Science, 311, 13011303.
Warneken, F., \& Tomasello, M. (2009). Varieties of altruism in children and chimpanzees. Trends in Cognitive Sciences, 13 (9), 397-482.

Wellman, H. (1990). The child's theory of mind. Cambridge, MA: MIT Press.

Wellman, H. (2010). Developing a theory of mind. In U. Goswami (Ed.), The Blackwell handbook of childhood cognitive development (2nd edn., pp. 258-284). Oxford: Blackwell.

Woodward, A.L. (1998). Infants selectively encode the goal object of an actor's reach. Cognition, 69, 1-34.

Received: 1 March 2010

Accepted: 15 July 2011 